Short Communication

\title{
Der Jesuit Athanasius Kircher und sein Organum mathematicum
}

Franz Daxecker

\begin{abstract}
Summary
P. Athanasius Kircher SJ (1602-1680) was a man of great erudition who carried out research in both the natural sciences and the humanities. He drew one of the first maps of the moon, performed microscopic studies of blood, worked on hieroglyphics and described China. He also invented the socalled Organum mathematicum. The device (located in Florence) has nine compartments containing wooden slats devoted to various mathematical subjects: arithmetic, geometry, fortifications, chronology, horography, astronomy, steganography (encryption) and music.
\end{abstract}

\section{Zusammenfassung}

P. Athanasius Kircher SJ (1602-1680) war ein Mann mit grosser Bildung; seine Forschungen beschäftigten sich mit Natur- und Geisteswissenschaften, er zeichnete eine der ersten Mondkarten, untersuchte das Blut unter dem Mikroskop, beschäftigte sich mit den Hieroglyphen und beschrieb China. Sein Organum mathematicum in Florenz enthält neun Fächer mit Stäben zu verschiedenen mathematischen Themen: Arithmetik, Geometrie, Fortifikation (Lehre vom Befestigungsbau), Chronologie, Horographie, Astronomie, Astrologie, Steganographie (Lehre von den Geheimschriften) und Musik.

Univ.-Prof. Dr. Franz Daxecker, Anichstrasse 35, A-6020 Innsbruck 


\section{Biographischer Abriss}

Athanasius Kircher wurde am 2. Mai (Athanasiustag) $1602^{1}$ als siebtes Kind und vierter Sohn von Johann Kircher und seiner Ehefrau Anna in Geisa bei Fulda geboren. Er trat am 2. Oktober 1618 in Paderborn in die Gesellschaft Jesu ein, wurde 1628 zum Priester geweiht und war ab 1629 Professor in Würzburg (Abb. 1). In seinen Forschungen beschäftigte er sich sowohl mit der Natur als auch mit den Geisteswissenschaften. Auf ihn geht das Organum mathematicum zurück, die erste gedruckte Karte der wichtigsten Meeresströmungen und eine der ältesten Mondkarten. Kircher führte als erster Blutuntersuchungen mit dem Mikroskop durch; er vermutete richtigerweise, dass kleine Lebewesen die Pest verursachen. Athanasius Kircher beschrieb die Laterna magica, er gründete in Rom das Museum Kircherianum, ferner beschäftigte er sich mit Vorarbeiten zu einer koptischen Grammatik und mit den Hieroglyphen; diese Vorarbeiten wurden vom ersten Entzifferer der Hieroglyphen, Champollion, benutzt. Er führte einen Briefwechsel mit über 700 Personen. Kircher meldete sich 1629 ohne Erfolg als China-Missionar. Sein erstes Werk Ars magnesia veröffentlichte er 1631. Im Jahr 1633 wurde er als Hofmathematiker Kaiser Ferdinands II. als Nachfolger Johannes Keplers nach Wien berufen (er trat diese Stelle nie an), im gleichen Jahr nach Rom als Professor für Mathematik, Physik und orientalische Sprachen im Collegium Romanum. 1635 machte er mehrere astronomische Beobachtungen und studierte mit Melchior Inchofer die Mondesfinsternis vom 27. bis 28. August. 1636 schrieb er das Werk Prodromus coptus sive aegyptiacus. Am 29. Juni 1637 legte er in Malta die Profess-Gelübde der Gesellschaft Jesu ab. 1641 erschien das Buch Magnes sive de arte magnetica in Rom, 1643 seine Lingua aegyptiaca restituta. Von seiner Lehrtätigkeit wurde Kircher 1645 befreit. 1650 begann ein Briefwechsel mit Herzog August d.J. von Braunschweig-Lüneburg Im selben Jahr entstanden die Musurgia universalis sive ars magna consoni et dissone und Obeliscus Pamphilius. Caspar Schott kam 1652 nach Rom und wurde Kirchers Schüler. Am 26. Dezember 1655 besuchte die Konvertitin Christina von Schweden das Collegium Romanum und traf oft mit Kircher zusammen. Beim ersten Zusammentreffen hiess Kircher sie in 34 Sprachen willkommen. 1659 war Kircher in Florenz bei den Medici; er trug viel zum Druck der Werke Galileis bei. 1661 erschien

1 Ich danke Frau Dr. Rita Haub, Leiterin des Archivum Monacense Societatis Jesu, dass sie mich auf dieses Thema aufmerksam gemacht hat und dass sie mir die Übersetzung von Caspar Schott (Organum mathematicum libris IX explicatum, Würzburg 1668) zur Verfügung gestellt hat (aus dem Lateinischen übersetzt von P. Alban Müller SJ). Aus dieser Übersetzung wurden Anregungen entnommen. Frau Dr. Haub hat auch darauf hingewiesen, dass in den Katalogen der Gesellschaft Jesu in Rom als Geburtsjahr 1601 angeführt wird. 


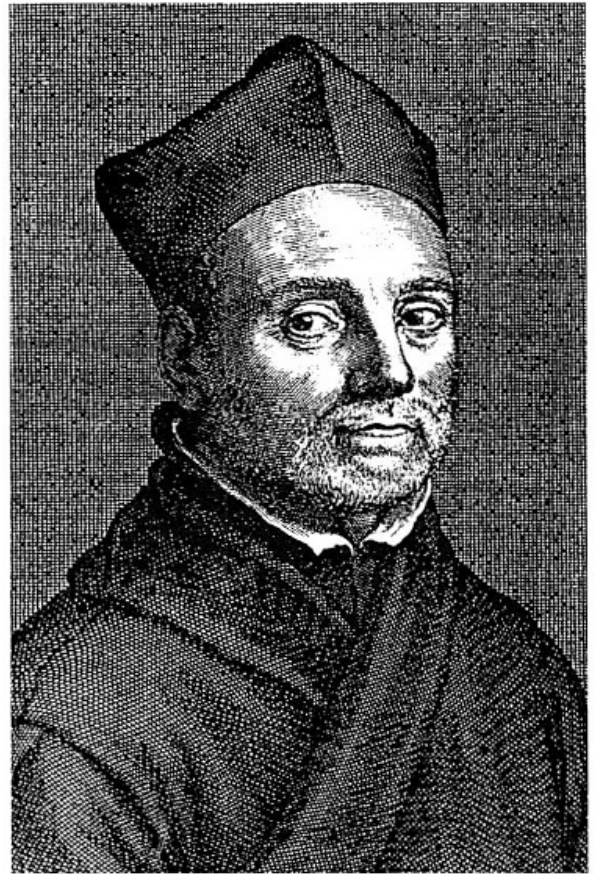

Abb. 1. P. Athanasius Kircher SJ.

die Diatribe de prodigiosis crucibus und 1663 die Polygraphia nova et universalis, ein Jahr später Mundus subterraneus I, der zweite Teil erschien 1665. Ein Jahr später publizierte er Obelisci aegyptiaci interpretatio. Das Werk China illustrata veröffentlichte er 1667 in Amsterdam und im selben Jahr Magneticum naturae regnum in Rom. 1668 erschien in Würzburg das Organum mathematicum. 1669 wurde in Amsterdam die Ars magna sciendi gedruckt. 1673 publizierte er die Phonurgia nova. Da er von vielen Besuchern bedrängt wurde, floh Kircher nach Mentorella, er wurde auch dort mit Briefen überhäuft. 1675 wurde in Amsterdam die Arca Noe gedruckt und 1676 Sphinx mystagoca sive diatribe hieroglyphica. In Amsterdam erschien 1679 Turris Babel sive archontologia und im selben Jahr Tariffa Kircheriana sive mensa Pythagorica expansa in Rom. Im Januar 1680 erkrankte er und starb am 27. November. Er wurde in der Kirche Il Gesù beigesetzt, sein Herz wurde in die Eustachius-Kirche in Mentorella gebracht ${ }^{2}$.

Athanasius Kircher war der Erfinder des Organum mathematicum (auch cista mathematica genannt), von dem noch mehrere Exemplare existieren. Eines befindet sich im Istituto e museo di storia della scienza in Florenz, dieses konnte der Autor im Frühjahr 1998 besichtigen ${ }^{3}$. Ein Organum mathematicum befindet sich in der Herzog-August-Bibliothek in Wolfenbüttel, zwei in Braunschweig ${ }^{4}$, eines im Bayerischen Nationalmuseum München.

2 Carlos Sommervogel, Bibliothèque de la Compagnie de Jésus, 9. Bde., Brüssel/Paris 1890-1900, Bd. 4, Sp. 1046-1077. - A. Behlau: Athanasius Kircher. Eine Lebensskizze, Heiligenstadt 1874. - Joscelyn Godwin, Athanasius Kircher. Ein Mann der Renaissance und die Suche nach verlorenem Wissen, Berlin 1994. - John Fletcher: «Athanasius Kircher Versuch eines Porträts», in: Universale Bildung im Barock. Der Gelehrte Athanasius Kircher. Ausstellungskatalog, Rastatt 1981, 15-17. - John Fletcher/Gerhard Römer, «Statt eines Lebenslaufs - Zeittafel zu Athanasius Kircher», in: Universale Bildung im Barock (s. oben), 17-30. - Olaf Hein/Rolf Mader, Athanasius Kircher S.J. in Malta, Berlin 1997.

3 Das Organum mathematicum befindet sich im Saal 1, Mathematikkästen, es ist als «Cista mathematica» unter der Inventarnummer 2741 registriert.

4 Mara Miniati, «Les cistae mathematicae et l'organisation des connaissances au XVII ${ }^{\mathrm{e}}$ siècle», in: Études sur l'histoire des instruments scientifices, ed. by Christine Blondel, Francoise Parot, Anthony Turner \& Mari Williams, London 1989, 43-51, 44, Anm. 5. 


\section{Das Organum mathematicum in Florenz}

Das Organum mathematicum ist ein Kästchen, das $25 \mathrm{~cm}$ tief, $29,5 \mathrm{~cm}$ breit, $44 \mathrm{~cm}$ hoch und durch einen pultartigen Deckel zu verschliessen ist. Nach dem Öffnen des Deckels werden senkrecht herausziehbare verschieden lange hölzerne Latten (Stäbe) sichtbar (Abb. 2), sie sind an der Vorder- und Rückseite mit Buchstaben und Zahlentabellen versehen. Zahnräder oder Wellen sind nicht vorhanden. Die Anwendung ist mit einem Rechenschieber vergleichbar. Je 24 Latten sind in neun nebeneinander stehenden Fächern mit verschiedenen mathematischen Themen angeordnet: Arithmetik, Geometrie, Fortifikation (Lehre vom Befestigungsbau), Chronologie, Horographie, Astronomie, Astrologie, Steganographie (Lehre von Geheimschriften), Musik. Auf dem pultförmig geneigten Deckel und auf der Vorderseite befinden sich zwei Kupferstiche.

\section{Zu den einzelnen Fächern ${ }^{5}$}

Erstes Fach: Arithmetik, dieses erste Fach mit 24 beidseitig beschrifteten Latten hat die Bezeichnung 1A und führt folgende Funktionen durch: Multiplikation, Division (ein Beispiel in der Fussnote) ${ }^{6}$, Quadratwurzelziehen, Kubikwurzelziehen. Die 24 Latten sind rosarot gefärbt.

Zweites Fach: Geometrie, bezeichnet mit dem Buchstaben 2B, es enthält Tabellen für die angewandte Geometrie, für die Vermessung von Schattenbildern des Quadranten und des geometrischen Quadrates und für die praktische Geometrie mit Hilfe arithmetischer Berechnungen. Die 24 Latten sind weiss coloriert.

5 Eine genaue Beschreibung ist durch den Schüler Caspar Schott erfolgt: Organum mathematicum libris IX explicatum, Würzburg 1668. Jesuiten aus Würzburg haben es nach Schotts Tod herausgegeben. Schott ist 1608 in Königshofen im Grubfeld geboren, gestorben ist er 1666 in Würzburg als Professor der Mathematik und Physik. Von ihm stammt eine Erklärung der Fata Morgana, er experimentierte in Würzburg an lebenden Tieren, indem er sie in luftleeren Raum brachte und infundierte Tieren Medikamente, z. B. Wein, der eine Berauschung hervorrief. P. Bernhard Duhr SJ, Jesuitenfabeln. Ein Beitrag zur Culturgeschichte, Freiburg 1899, 264.

6 Als Beispiel die Anleitung zu einer Division: «360 soll durch 6 geteilt werden. Gehe in folgender Weise vor: nimm den Teiler 6 in der mit dieser Zahl bezeichneten Tabelle und siehe zuerst wie oft 6 in 36 enthalten ist. Du findest die Tabelle hinabgehend 36, sie besetzt die 6. Stelle. Schreibe also 6. Dann nimm den Teiler und sieh nach, wie oft er in der vorhin hingeschriebenen Zahl [6] enthalten ist: es ergibt sich eine Null, schreibe die Null neben die 6, es entsteht der Quotient 60. So oft ist 6 in 360 enthalten.» Aus: Caspar Schott, Organum mathematicum libris IX explicatum, Würzburg 1668, erstes Buch Arithmetik, Kapitel II: Division, § II, p. 71. 
Drittes Fach: Fortificatoria (Festungsbau), bezeichnet in der Überschrift mit dem Zusatz 3C. Die Latten sind an der Spitze grün gefärbt und nur an der Vorderseite beschriftet. Dieses Fach enthält Anweisungen und Tabellen für den Festungsbau; es werden Vielecke mit Winkeln beschrieben (vom Viereck bis zum Zehneck) zur Berechnung von Wällen, Brustwehren, Gräben und offenen Wegen.

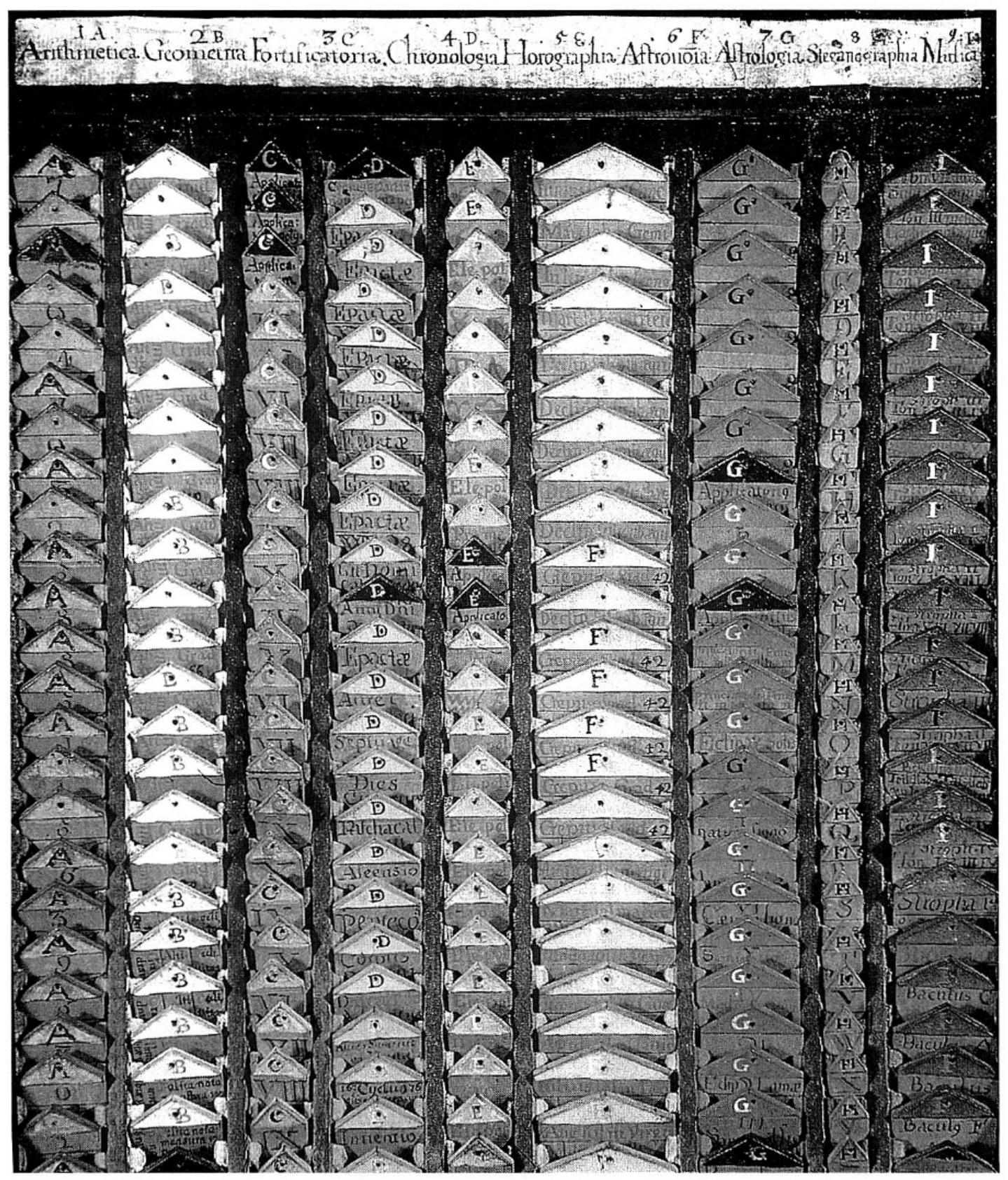

Abb. 2. Das geöffnete Organum mathematicum mit neun Reihen zu je 24 verschiedenfarbigen nach vorne zu kürzer werdenden hölzernen Latten, überschrieben mit: Arithmetica, Geometria, Fortificatoria, Chronologia, Horographia, Astronomia, Astrologia, Steganographia, Musica. 
Viertes Fach: Chronologia (Zeitberechnung, 4D), die Latten sind an der dreieckigen Spitze gelb gefärbt mit Ausnahme zweier, die schwarz gefärbt sind, und mit dem Buchstaben D bezeichnet. Sie haben verschiedene Einheiten wie Minuten, Stunden, Tag, Woche, Monat, Jahr, Feste, Zyklen und Perioden. Mit ihnen können die kirchlichen Zeiten bestimmt werden, der «Computus», zum Beispiel das Osterfest und andere bewegliche und unbewegliche Feste. Dieses Fach enthält in der Beschreibung von Caspar Schott noch vier Kreisscheiben für die Berechnung des Sonnenzyklus, der Schalttage, für die Festlegung des Osterfestes und für die Bestimmung des Indiktionszyklus (15-Jahre-Zyklus). Es können auch die astronomische Sonnenzeit und ihre Unterteilung in Stunden, Tage und Jahre berechnet werden, ebenso die astronomische Mondzeit. So wird zum Beispiel berücksichtigt, dass ein Jahr 365 Tage und 6 Stunden lang ist und dies in vier Jahren 24 Stunden ausmacht, aus diesem Grunde besteht jedes vierte Jahr aus 366 Tagen (Schaltjahr).

Fünftes Fach: Horographia (Uhrenkunde, 5E), diese Wissenschaft beschreibt den Bau von horizontalen oder vertikalen Sonnenuhren anhand von einigen fundamentalen Regeln. In diesem Fach sind wieder 24 Latten, 22 an der Spitze blau gefärbt und zwei schwarz gefärbt (E), letztere geben die Position von $42^{\circ}$ (Rom) und $38^{\circ}$ (Palermo) wieder, ferner ist noch Wien mit $48^{\circ}$ bezeichnet.

Sechstes Fach: Astronomie. Dieses Fach ist noch mit $6 \mathrm{~F}$ bezeichnet. Es enthält 24 Latten, deren Spitzen weiss gefärbt sind. Es lassen sich die Länge der Tage und Nächte für das ganze Jahr berechnen, die Stunden des Sonnenaufgangs und -untergangs, die Tierkreiszeichen, die Dämmerungszeiten für das ganze Jahr, die Höhe und die Neigung der Sonne.

Siebtes Fach: Astrologie, es ist noch mit dem Buchstaben $7 \mathrm{G}$ überschrieben, die Latten sind an der Spitze hellrot bemalt, zwei von ihnen sind an der Spitze schwarz, der Buchstabe $\mathrm{G}$ ist blau, weiss, schwarz oder gelb. Das astrologische Fach beschreibt die Vorauskenntnis oder Vorhersage aus den Sternen, die Eigenschaft der Sterne, die Bewegungen, das Zusammentreffen und ihr Aussehen. Die Astrologie wird daher als Seherin, Wahrsagerin und Entscheiderin bezeichnet. Das siebte Fach enthält vier Klassen von Tabellen mit den Zeichen für Saturn, Jupiter, Mars, Kopf und Schwanz des Drachens und die Knotenpunkte des Mondes. Ferner eine Einteilung in Grade, Minuten und Zeichen, ebenso die Tierkreiszeichen, die Stellung der Planeten und Knotenpunkte.

Achtes Fach: Steganographia (Geheimschriftkunde), dieses Fach ist zusätzlich oben noch mit $8 \mathrm{H}$ beschriftet. Die Steganographie ist die Wissenschaft von der Geheimschrift und ist den Monarchen, den Königen, Fürsten 
und Heerführern von Nutzen, ohne sie kann das ihnen anvertraute Amt kaum ausgeübt werden. Das Fach enthält 24 Buchstaben des deutschen Alphabets, auch das W. Diese Buchstaben sind den Buchstaben anderer Alphabete (z. B. lateinisch, griechisch, englisch, polnisch und belgisch) gleich; mit Hilfe eines Schlüsselsatzes kann ein beliebiger Brief in jede andere Sprache chiffriert werden.

Neuntes Fach: Musica (Musikkunde, 9I). Die 24 Latten sind an der Spitze violett, der Buchstabe I, der sich dort befindet ist weiss, blau, schwarz, rot oder gelb, sie enthalten Tabellen und Zahlen, die zur Komposition dienen; mit ihnen können Noten, Zeit, Tonart, Modulation und Dauer der Noten selbst bestimmt werden. Ebenso ist es möglich auf vorgegebene Versmasse einen Kontrapunkt zu komponieren.

Dieses Organum mathematicum wurde 1661 von Athanasius Kircher dem Erzherzog Karl Josef in Wien übersandt?

Über Athanasius Kircher gibt es viel Literatur ${ }^{8}$, über das Organum mathematicum wenig'; es wurde von Caspar Schott in lateinischer Sprache ausführlich beschrieben ${ }^{10}$, die Beschreibung ist schwer zugänglich. Dem Organum mathematicum liegen die Neperianischen ${ }^{11}$ Tafeln zugrunde; als Vorläufer eines mechanischen oder elektronischen Computers kann es nicht bezeichnet werden. Caspar Schott hat in Anlehnung an das Organum mathematicum eine mechanische Rechenmaschine entwickelt, den sogenannten Schottschen Rechenzylinder ${ }^{12}$.

7 Karl Josef, Sohn von Kaiser Ferdinand III. und Erzherzogin Maria Leopoldine, 2. Ehe (geb. 7.8.1649 in Wien, gest. 27.1.1664 in Linz, begraben in der Kapuzinergruft in Wien), er erhielt bereits als Elfjähriger die Tonsur, er sollte Bischof von Passau werden, starb aber im 15. Lebensjahr. Brigitte Hamann, Die Habsburger. Ein biographisches Lexikon, Wien ${ }^{3} 1988$, 210.

8 Siehe Anm. 2.

9 Siehe Anm. 4 und 12.

10 Siehe Anm. 5.

11 John Napier (Neper) (1550-1670), entwickelte siebenstellige Logarithmen, als weiteres Hilfsmittel erfand er die Neperschen Stäbchen für mechanische Berechnungen.

12 Peter Friess/Eva Langenstein, Mechanik aus der Wunderkammer-Die Vorläufer der Computer, München 1996, 38-39. 